\title{
Ueber einen Fall von Graves'scher Krankheit mit Exophthalmus monocularis und einseitiger Schilddrüsen-Anschwellnng ${ }^{1}$ ).
}

\author{
Yon \\ Dr. Percy Fridenberg \\ in New York.
}

Hierzu Tafel IX.

Seit der Zeit als die klassisch gewordenen Vorlesungen des Dubliner Klinikers zuerst die Aufmerksamkeit auf den Symptomencomplex von Kropfbildung, Herzklopfen und anämischer Hervorragung der Augäpfel lenkten und Basedow wenige Jahre später und unabhängig dieselben Symptome als charakteristische Erscheinungen einer bestimmten "Glotzaugen-Cachexie" zusammenstellte, ist wohl keine Theorie aufgestellt worden, welche die Pathologie und Aetiologie dieser interessanten Affection in vollständig befriedigender Weise erklärt, obwohl die Graves'sche oder Basedow'sche Krankheit ein ganz bestimmtes und gar nicht seltenes klinisches Wesen darstellt. Bei einem solchen Stande unseres Wissens iuber diesen Gegenstand dürfte die Mittheilung eines Falles von Graves'scher Krankheit mit der seltenen Erscheinung von Beschränkung der Augensymptome auf

1) Vortrag gehalten in der Section on Ophthalmology, New York Academy of Medicine, 18. März 1895. 
eine Seite, verbunden mit einer Hypertrophie des contralateralen Schilddrïsenlappens nicht ohne Interesse sein.

Die Patientin, welche diese interessante Variation darbot, stellte sich an 3. November 1894, asthenopischer Beschwerden wegen im New Yorker Eye and Ear Infirmary vor und wurde mir zur Feststellung des Refractionszustandes übergeben. Meinem verehrten Chef, Dr. E. Gruening, verdanke ich die Erlaubniss, folgende Beobachtungen mitzutheilen.

Die Patientin, Frau A. J., ist 24 Jahre alt. Vater und Mutter, 54 bezw. 48 Jahre alt, sind rüstig und gesund. 6 Brïder und 4 Schwestern anscheinend kräftig und wohl, leiden nach den Aussagen der Patientin an nervöser Schwäche. Die Patientin giebt an, immer gesund gewesen zu sein, obwohl seit Kindheit nervös, schüchtern, und ängstlich. Sie ist seit vier Jahren verheirathet. Vor drei Jahren erfolgte, im vierten Monate der Schwangerschaft, ein Abortus, und eine zweite Schwangerschaft ist nicht eingetreten. Seit der Eheschliessung hat die Patientin durch unglückliche Familienverhältnisse zu leiden gehabt; vor ungefähr 6 Monaten wurde ihr Zustand der Reizbarkeit noch durch ein besonders peinliches Ereigniss verschlimmert. Binnen einigen Wochen bemerkte sie eine ungewöhnliche Ermüdbarkeit nach einer, wenn auch noch so geringen Anstrengung, besonders nach dem Gehen und dem Treppensteigen, verbunden mit häufiger Röthung der Gesichtshaut und profusen Schweissen. Die nervöse Reizbarkeit nahm merklich zu, mit Herzklopfen und Athembeschwerden, und zuweilen mit dem Gefühl von Klopfen und Schwirren in den Halsgefässen. Die Patientin, eine Näherin, klagte über Schmerzen in den Augen und über eine $\mathrm{Ab}$ nahme der Sehschärfe, wenn sie auch nur kurze Zeit nähte. Man sagte ihr, sie sehe "wild" aus, und "das eine Auge sei grösser als das andere." Noch ehe ich die Einzelheiten der Krankengeschichte erfahren hatte, lenkte der eigen- 
thümliche Ausdruck der linken Gesichtshälfte meine Aufmerksamkeit auf den unheimlich starren Blick des linken Auges. Dieses charakteristische Merkmal, welches in der ungefähr zwei Monate nachher aufgenommenen Photographie sehr deutlich hervortritt, liess mich die Diagnose vermuthen. Die linke Lidspalte war durch eine merkliche Retraction des Oberlides erweitert, und bei geradeaus gerichtetem Blicke sah man oberhalb des Cornealrandes die weisse Sclera in einer Ausdehnung von $2 \mathrm{~mm}$ frei liegen. Der Augapfel war merklich, aber nicht hochgradig prominent; das Graefe'sche Symptom liess sich mit Leichtigkeit demonstriren. Der unwillkürliche Lidschlag war in seiner Häufigkeit vermindert, aber nicht aufgehoben. Die Sehschärfe war beiderseits normal; die Refraction emmetropisch. Eine Insufficienz der inneren geraden Augenmuskeln von $2^{0}$, resp. von $5^{0}$, wurde für die Nähe und für die Ferne constatirt. Bei der ophthalmoskopischen Untersuchung sah man links Pulsation der Venen auf der Papille, aber keinen Arterienpuls. Das rechte Auge zeigte nichts Abnormes; der Ausdruck dieser Gesichtshälfte war ein ganz natürlicher. Das Gesicht war geröthet, besonders die linke Seite, und diese Erscheinung nahm bei der leichtesten psychischen Erregung in hohem Grade zu. Die Schilddrüse war nicht merklich vergrössert; bei der Palpation aber fühlte man eine deutliche Schwellung des rechten Lappens und des Isthmus. Es zeigte sich ein feiner, fast fibrillärer Tremor der Hand und der Zunge, ähnlich dem bei Paralysis agitans beobachteten, aber etwas langsamer, 5-6 mal in der Secunde. Der Herzschlag war kräftig und hebend, zuweilen aussetzend; der zweite Pulmonalton verstärkt. Zeichen eines Klappenfehlers fehlten vollständig, aber die Herzdämpfung war nach links vergrössert, der Spitzenstoss diffus. Ein deutlicher Capillarpuls war an der Brust und am Halse zu bemerken. An der Vena jugularis hörte man lautes Nonnensausen, und über dem vergrösserten rechten Schild- 
drüsenlappen ein blasendes Geräusch, synchron mit dem ersten Herztone. An den Lungen war nichts Abnormes, aber die Inspiration oberflächlich und zuweilen keuchend; der Thorax etwas flach. Die Carotiden pulsirten beiderseits sehr kräftig; besonders links, aber der Radialpuls war klein und weich, 120 in der Minute, bei ruhendem Körper. Obwohl ich der Patientin die Wichtigkeit der geistigen und körperlichen Ruhe und die voraussichtliche Verschlimmerung der Symptome bei einer Vernachlässigung der Behandlung einschärfte, vergingen doch einige Wochen, ehe sie sich einer consequenten Therapie unterzog. Es wurde bei Bettruhe eine Regulirung der Diät eingeleitet, und bei Lästigwerden der Palpitationen das Auflegen eines Eisbeutels auf das Präcordium und auf die vergrösserte Schilddrüse empfohlen. Ich verschrieb neben einem, Ferrum, Strychnin. sulphur. und Chinin enthaltendem Tonicum, sechs Tropfen einer Digitalis-Tinctur dreimal täglich. Die Herzaction wurde durch letzteres Mittel gar nicht verbessert, obwohl die Dosis im Laufe der nächsten 3 Wochen bis auf 20 Tropfen viermal täglich erhöht wurde, und auch die Eisbeutel, welche der Patientin sehr angenehm waren, schienen in dieser Beziehung keinen genïgenden Einfluss zu haben. Die Digitalis wurde deshalb ausgesetzt, und die von Fraser so warm empfoblene Tinctura Strophanthi in Dosen von 6 Tropfen dreimal täglich verabreicht. Jeden Abend bekam die Patientin $1 \mathrm{~g}$ Bromkalium. Der Fall ist jetzt seit 6 Monaten unter meiner Beobachtung, und der Zustand hat sich entschieden gebessert. Der Herzschlag ist weniger schnell; die Palpitationen, obwohl noch zuweilen vorhanden, weniger lästig. Die Protrusion des linken Auges ist fast nicht mehr zu erkennen; die Retraction des Oberlides hat merklich abgenommen. Die Besserung im Ausdruck der Patientin wurde von ihrer Umgebung gerïhmt, was auf den psychischen Zustand der ersteren einen günstigen Einfluss gehabt zu haben scheint. Die Prognose dürfte in Bezug จ. Graefe's Archiv für Ophthalmologie. XLI. 3. 
auf eine dereinstige vollkommene Genesung ziemlich günstig sein.

In diesem Falle waren die klassischen Symptome der Graves'schen Krankheit nebst anderen vorhanden, welche, wenn auch nicht pathognomonisch, doch für diese Affection charakteristisch sind. Von diesen erwähne ich den Tremor (Marie), die nervöse Schwäche und Reizbarkeit (Charcot), dje Insufficienz der Interni (Möbius), die oberflächliche Respiration (Bryson) und die Röthung und Hyperhidrose der Haut mit der begleitenden Verminderung des Widerstandes gegen den galvanischen Strom. Die merkwürdigste Erscheinung in diesem Falle ist aber unbedingt die ungewöhnliche Beschränkung der Augensymptome auf die linke Seite, verbunden mit einer "gekreuzten" Hypertrophie des rechten Schilddrïsenlappens. Einseitige Symptome sind nach den meisten Theorieen der Krankheit so unerklärlich, dass einige Autoren ${ }^{1}$ ) das Vorkommen einer derartigen Variation geleugnet haben, während Berger ${ }^{2}$ ) das Vorhandensein von einseitigem Exophthalmus als entscheidenden Beweis gegen die Diagnose von Graves'scher Krankheit ansieht. Es lässt sich gar nicht bezweifeln, dass in vielen Fällen, die als Morbrus Basedowii s. Gravesii veröffentlicht wurden, besonders aus den ersten Jahren der Beobachtung auf diesem Gebiete, die Diagnose unrichtig war. Affectionen des Sympathicus-Grenzstrangs mit atypischen Symptomen ${ }^{3}$ ), complicirte Klappenfehler ${ }^{4}$ ), Erscheinungen hysterischer Art bei chlorotischen Mädchen und, in einigen Fällen, wahrer endemischer oder auch sporadischer Kropf mit einseitigen Drucksymptomen ${ }^{5}$ ). wurden alle in die bequeme Kategorie

) Schott, Deutsehe med. Zeitung Nr. 32, 1889. Marcus, ibid. Nr. 48, 1893.

2) Bull. de la Soc. de Chir., p. 277. 1884.

3) Chvostek, Wiener med. Presse, p. 497. 1872.

4) Schnitzler, Wiener Med.-Halle, Nr. 24, p. 245, 1864.

5) Berger, Bull. de la Soc. de Chir., p. 277. 1884. 
des Morbus Basedowii hineingeworfen. In der LiteraturZusammenstellung welche L. Hirschberg ${ }^{1}$ ) seiner historisch-kritischen Studie beigegeben hat, finden sich mehrere Fälle, die beim Nachschlagen der Originalartikel sich als gewöhnliche "bilaterale" entpuppen ${ }^{2}$ ), andere, in denen ein mehr oder weniger ausgesprochener Unterschied in dem Grade des Exophthalmus vorhanden war $^{3}$ ), während in einer dritten Serie die Beschränkung der Symptome auf eine Seite nur eine voribbergehende war, indem die Protrusion an dem einen Auge etwas früher als an dem anderen auftrat ${ }^{4}$ ).

Mit Ausschluss der zweifelhaften Fälle finden sich folgende Beobachtungen auf diesem Gebiete. Demours ${ }^{5}$ ) sah bei einem 11 Jahre alten Mädchen eine Prominenz des linken Auges von ungefähr 2 Linien, welche 3 Jahre persistirt hatte. Die Patientin hatte seit Kindheit eine leichte Vergrösserung der Schilddrüse. Die Mutter zeigte auch eine Disposition zur Kropfbildung, und nach der ersten Schwangerschaft stellte sich eine Schilddrüsenhypertrophie ein, welche stetig zunahm. Desmarres ${ }^{6}$ ) publicirte unter dem Titel "De l'exophthalmie produite par l'hypertrophie du tissu cellulo-adipeux de l'orbite" mehrere Fälle Graves'scher Krankheit. In einem Falle wurde bei einem 30jährigen Weibe, welches an nervöser Reizbarkeit, Herzklopfen und Schilddrüsen-Hypertrophie litt, ein einseitiger Exophthalmus leichten Grades, und zwar des rechten Auges be-

1) Wiener Klinik, 1894. Die Basedow'sche Krankheit.

2) Rosenberg, Berl. klin. Wochenschrift. 1865, II. p. 277. Sichel, Bull. gén. de Therap. Tome XXX. 1853.' Patchett, Lancet, 1872. p. 827.

3) Emmert, v. Graefe's Archiv XVII. 1. p. 203. XVII.

4) Jendrassik, Archiv f. Psychiatrie und Nervenkrankh. 1863.

5) Traité des Maladies de l'oeil. Paris 1818.

๑) Gaz. des Hôpitaux, 1853. Nr. 1, p. 2. 
P. Fridenberg.

obachtet. Das obere Lid war retrahirt, die Motilität vollkommen; die Sehschärfe normal. Nach Behandlung mit Jodkalium und örtlicher Application einer Jodsalbe verbesserte sich der Zustand erheblich. In seiner allgemeinen Zusammenfassung beschreibt Desmarres klar die eigenthümliche Physiognomie der Basedow-Kranken und macht besonders auf ein Symptom aufmerksam, welches Stellwag entdeckt haben soll, 6 Jahre nachdem Desmarres dasselbe folgendermassen beschrieben hatte: "Lorsqu'on regarde attentivement un malade atteint de cette affection, on remarque, comme premier symptome, que la paupière supérieure ne s'abaisse plus comme dans l'état physiologique sur la partie supérieure de la comée, lorsque l'oeil regarde horizontalement; au contraire, la comée reste découverte en totalité, ce qui donne à la physiognomie quelque chose de hagard fort désagréable à voir. A un dégré un peu plus avancé encore le malade a les yeux d'un homme en fureur, et cela établit un contraste choquant avec la tranquillité du reste de la physiognomic."

Mackenzie ${ }^{1}$ ) sah einen Fall. Seine Patientin, eine schwächliche, anämische junge Frau, deren Schwester seit Jahren wegen Graves'scher Krankheit in Behandlung war, litt an Amenorrhoe mit nervösen Symptomen, Verdauungsbeschwerden und Verstopfung. Nach einigen Monaten psychischer Erregung und körperlicher Anstrengung während des Sommers fing das rechte Auge an zu prominiren, und die Schilddrüse vergrösserte sich. Die Symptome gingen zurück nach dem Gebrauch ron Tinctura Jodi (Dosis nicht angegeben), und Einreibungen von Jodkaliumsalbe am Halse und in der' Schläfengegend.

Praëla) beobachtete die Entwicklung eines rechtsseitigen Exophthalmus mit bilateralem Kropfe in der Convalescenz nach einer acuten Bronchitis, bei einem 50jährigen

1) Pract. on the Diseases of the Eye, Ed. IV. 1854.

2) v. Graefe's Archiv III. 2. p. 199. 
Manne, welcher seit dem 20. Jahre an Palpitationen und anderen Zeichen einer Herzaffection gelitten hatte. Nach langer Zeit fing das linke Ange auch an $\mathrm{zu}$ prominiren. Der Fall endigte letal und bei der Section fanden sich ausgedehnte atheromatöse Veränderungen an der Aorta, mit einer Insufficienz und Stenose der Mitralis. In zwei anderen Fällen wurde rechtsseitiger Exophthalmus bei chlorotischen Mädchen von 19 resp. 15 Jahren constatirt. In dem einen Falle stellten sich später Herzklopfen und profuse Schweisse ein und hielten mehrere Jahre lang an. Im anderen Falle ging ein schwerer psychischer Insult der Krankheit voraus. Der Exophthalmus nahm fünf Jahre später plötzlich zu, nachdem ein schwerer Anfall von Blutbrechen einen Zustand acuter Anämie herbeigeführt hatte. Ein Kropf wurde jetzt zum ersten Male bemerkt.

Chvostek ${ }^{1}$ ) sah einen Fall bei einer 55jährigen Frau. Die Mutter war seit Kindheit nervös und schwächlich. Der Vater litt an einem Herzklappenfehler, und eine Schwester zeigte seit 25 Jahren ausgesprochene Symptome der Graves'schen Krankheit. Die Patientin' hatte vor 8 Jahren eine starke psychische Erregung gehabt, und bald nachher stellten sich die klassischen Symptome, aber mit vorwiegend einseitigen Erscheinungen ein. Das rechte Auge war deutlich prominent; die rechte Thyreoidea sehr vergrössert. Hyperhidrose, Röthung der Haut und hochgradige Abmagerung, alle auf die rechte Seite beschränkt, kamen später hinzu und der Allgemeinzustand wurde ominös. Trotzdem genas die Kranke im Laufe eines Jahres vollkommen.

Einen merkwürdigen Fall theilt Burney $\mathrm{Yeo}^{2}$ ) mit. Die Patientin, eine 35 Jahre alte Frau, war bis zur vierten Entbindung immer gesund, obwohl nervös und reizbar. Im Puerperium stellten sich die Erscheinungen einer allgemei-

$\left.{ }^{2}\right)$ Wiener Med. Presse. 1872, p. 497.

2) Brit. Med. Journal. Mch. 17, 1877. 
nen Sepsis ein, welche drei Wochen dauerten. Als die Kranke wieder aufstehen durfte, wurde ihr von der Umgebung nicht gestattet, sich im Spiegel zu betrachten, "weil sie so wild aussehe". Das linke Auge prominirte hochgradig, das rechte war in seiner normalen Stellung geblieben. Der rechte Lappen der Gl. thyreoidea war deutlich vergrössert, der linke wenig oder gar nicht verändert. Zur selben Zeit fingen die Wimpern und die Haare der Augenbraue links an auszufallen. Psychische Unruhe, Röthung der Haut, Hyperhidrose und Herzklopfen mit einem Pulse von 136 in der Minute, waren vorhanden. Kropf und Exophthalmus waren deutlich unilateral, aber "gekreuzt". Nach 6 Monaten wurde das rechte Auge auch prominent, und auch an diesem Auge wurde das merkwürdige Symptom der Alopecie der Wimpern und der Augenbraue beobachtet. Gleichzeitig fing der gegenüberstehende Schilddrüsenlappen an, sich zu vergrössern, und erreichte bald den Umfang des rechten. Yeo erwähnt einen zweiten Fall von einseitigem Exophthalmus, welcher bei einem früher gesunden und kräftigen 23jährigen Mädchen seit einem Jahre bestand. Später kamen Herzklopfen und nervöse Symptome hinzu; der Puls schwankte zwischen 136 und 140, aber eine Vergrösserung der Schilddrüse liess sich nicht constatiren.

Becker ${ }^{1}$ ) behandelte eine Dame von 28 Jahren, welche 7 Jahre verheirathet und Mutter eines kräftigen, gesunden Knaben war. Eine zweite Schwangerschaft war nicht eingetreten, da Dysmenorrhoe mit ausgesprochenen nervösen Symptomen der Geburt gefolgt waren, welche schliesslich in einen Zustand wahrer Hysterie ausarteten. Seit einem Jahre klagte sie iber Herzklopfen und bemerkte zuweilen eine Prominenz des linken Auges, während das rechte vollkommen normal blieb. Die Schilddrüse war leicht ver-

1) Klin. Monatsbl, f. Augenheilk. XVIII. 1880. 
grössert; die Sehschärfe normal. Am linken Auge Arterienpuls an der Papille und Exophthalmus mässigen Grades. Das rechte Auge zeigte nichts Abnormes.

Abadie ${ }^{1}$ ) beschreibt folgenden Fall. Eine junge Dame, 28 Jahre alt, welche seit einigen Monaten an hochgradiger Ermüdbarkeit, allgemeinem Unbehagen, Herzklopfen und Nervosität litt, wurde eine Zeit lang auf Anämie behandelt. Die Diagnose hatte man per exclusionem gestellt, da die klinische Untersuchung keine organischen Abnormitäten erwies. Später bemerkte die Patientin eine Prominenz des rechten Auges und stellte sich in der Abadie'schen Klinik vor. Der charakteristische Ausdruck des Auges, durch die Retraction des oberen Lides bedingt, liess sogleich die wahre Natur des Falles vermuthen. Der Puls war bei ruhendem Körper 96 in der Minute. Die Prominenz des rechten Auges war evident. Das linke Auge sah vollkommen normal aus. An der Schilddrise wurde eine Vergrösserung des Isthmus leichten Grades festgestellt.

Bei einem 24 Jahre alten Manne, welcher 3 Jahre lang an Palpitationen gelitten hatte, fand Maher ${ }^{2}$ ) eine besonders am rechten Lappen vergrösserte Schilddrüse und rechtsseitigen Exophthalmus. Auf dieser Seite prominirte der Hornhautscheitel 2-3 mm. Dieser Zustand wurde von dem Patienten seit einem Jahre beobachtet. Das Graefe'sche Symptom war nicht vorhanden.

In Amerika ist kein Fall von Graves'scher Krankheit mit einseitigen Symptomen publicirt und meines Wissens nur einer beobachtet worden. Dieser Fall wurde am 22. December 1893 in der wissenschaftlichen Zusammenkunft deutscher Aerzte von Dr. George W. Jacoby vorgestellt, welcher mir freundlichst seine Daten nebst einer Photographie der Patientin zur Verfügung gestellt hat.

I) L'Union médicale 1880, Nr. 157. p. 859.

2) Lancet, 1886, Nr. I. p. 1221. 
168 P. Fridenberg. Ueber einen Fall von Graves'scher Krankheit etc.

Kate, P., 33 Jahre alt, klagt seit ungefähr 2 Monaten iber allgemeine nervöse Symptome, Herzklopfen und Schweisse. Seit derselben Zeit bemerkt sie ein fremdes Aussehen des rechten Auges mit einer Prominenz des Bulbus. Bei der Untersuchung zeigt sich Retraction des oberen Lides mit Exophthalmus mässigen Grades und das Graefe'sche Symptom, alles am rechten Auge. Insufficienz der Interni (Möbius) war vorhanden; der unwillkürliche Lidschlag war nicht vermindert. Die rechte Hornhaut war vielleicht etwas anästhetisch. Die Schilddrüse war etwas vergrössert, der rechte Lappen entschieden voller als der linke. Neben diesen Symptomen war Tremor und bronzeartige Verfärbung der Haut vorhanden.

In diesen 13 Fällen war das rechte Auge nur dreimal afficirt. Von diesen 3 Fällen war nur einer, der von Burney $\mathrm{Yeo}$, mit einer Hypertrophie des contralateralen Schilddrüsenlappens vergesellschaftet, und auch in diesem Falle wurden schliesslich beide Lappen hypertrophisch. Rechtsseitiger Exophthalmus mit rechtsseitigem Kropfe war nur in dem einen, von Chrostek mitgetheilten Falle vorhanden. In meinem Falle ist bisher kein Zeichen eines Weitergreifens der Affection auf die andere Seite bemerkt worden und die deutliche Abnahme der Symptome berechtigt mich zu der Hoffnung, dass der gegenwärtige gekrewzte Zustand schliesslich in Heilung übergehen wird, ohne dass irgend welche Veränderung in der auffallenden Beschränkung des Exophthalmus und des Kropfes eingetreten wäre. 
v. Graefe's Archiv Bd. X7.1, 3.

Taf. IX.

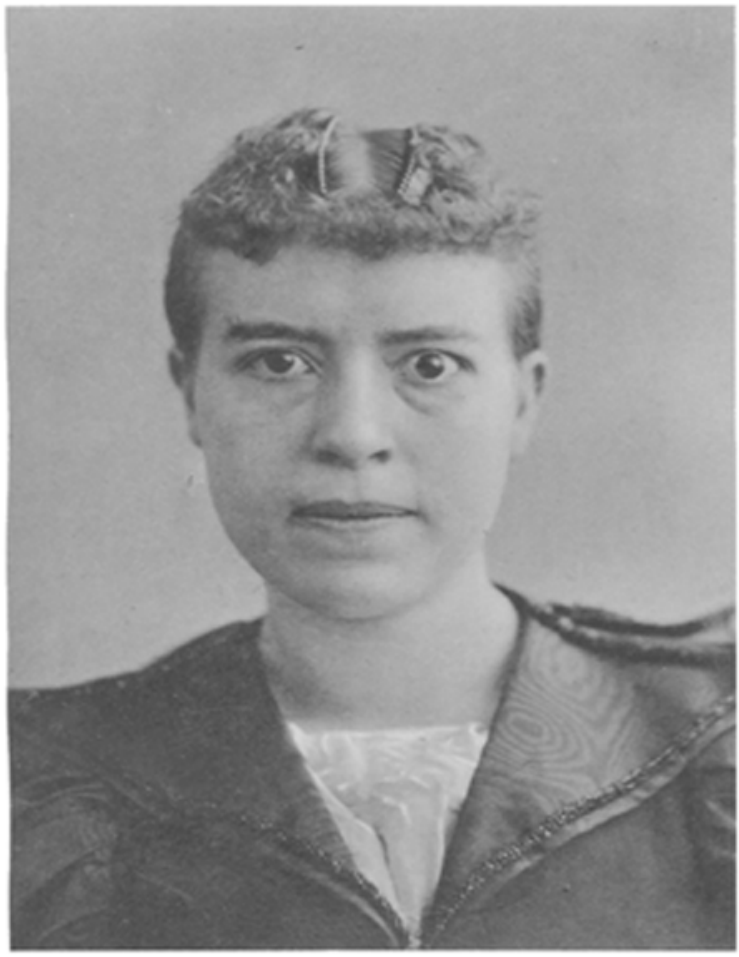

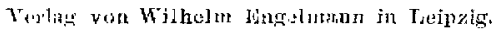

